\title{
METAPHASE KARYOTYPES OF CERTAIN SPECIES OF THE DROSOPHILA MONTIUM SUBGROUP
}

\author{
VISUT BAIMAI \\ Department of Biology, Faculty of Science, Mahidol University, \\ Rama 6 Road, Bangkok 4, Thailand
}

Received January 31, 1980

\begin{abstract}
A total of 20 species in the Drosophila montium subgroup has been cytologically examined. All species show similar basic pattern of metaphase karyotype. The most extensive variations in metaphase chromosome configurations have been observed in the $\mathrm{Y}$ and 4 th (dot) chromosomes while the $\mathrm{X}$ chromosome is slightly variable. Interspecific karyotype differentiation is largely due to the acquisition of different amounts of heterochromatin.
\end{abstract}

\section{INTRODUCTION}

The Drosophila montium subgroup, belonging to the melanogaster species group of the genus Drosophila, comprises some 58 described species (Bock and Wheeler 1972; Bock, personal communication). Almost all species of this subgroup are characterised by a large sex-comb on the male foreleg. Most of the members of the subgroup are very similar in external morphology. Classification of the species is essentially based on the structure of male genitalia, which are quite distinct in most cases but indistinguishable in some sibling species complexes. Two such sibling species of the $D$. kikkawai complex from the Southeast Asian region have recently been described based on the evidence of cytogenetic studies (Tsacas and David 1977; Baimai 1979).

Some information on metaphase karyotypes of 22 species of the montium subgroup has been compiled in an extensive catalog recently prepared by Clayton and Wheeler (1975). There are only two species of this subgroup that have been extensively investigated cytologically, i.e. D. birchii and D. kikkawai (Baimai 1969a, 1969b, 1978; Baimai and Chumchong 1980). These two species strikingly exhibit intraspecific variation in metaphase chromosome configurations which is mainly due to the different amount of extra heterochromatin particularly involving the sex chromosomes and the small 4th chromosome. Although metaphases of members of the montium subgroup had been included in some cases in the species descriptions, detailed accounts and photographs of the metaphase chromosome configurations suitable for cytological comparisons have been largely lacking. The aim of this study is to reinvestigate metaphase karyotypes of certain species of the montium subgroup available in the laboratory to determine: (a) details of chromosome configurations, e. g. the amount of extra heterochromatin 
and secondary constrictions, (b) interspecific variations of metaphase karyotypes, and (c) possible intraspecific variations in addition to those of the two species already known. This is a report on recent findings and interpretations regarding metaphases of some members of this interesting subgroup of the genus Drosophila.

\section{MATERIALS AND METHODS}

Most of the laboratory culture stocks of the montium species subgroup available for this study were kindly provided by The Genetics Foundation, University of Texas at Austin. Two newly described species were collected from wild populations in Thailand by the author and his colleagues (Table 1).

Preparations of metaphase karyotypes were made from 3rd instar larval brain ganglions using the standard method of Lewis and Riles (1962) and the technique recently described by Baimai (1977), consisting essentially of pretreatment of the brain ganglion by $0.1 \%$ colchicine solution or $10 \mathrm{mcg} / \mathrm{ml}$ of colcemid in Hanks' Balanced Salt Solution (Gibco) before proceeding to the routine lactic-acetic-orcein squash preparations or heatdry method. These procedures yielded a large number of well-spread metaphase chromosomes favourable for detailed analysis and photographic comparison. Photomicrographs of metaphase chromosomes were taken by Kodak High Contrast Copy Film under oil immersion (670 magnification) with green filter.

\section{RESULTS AND DISCUSSION}

The results of this study revealed that there are no major changes in metaphase karyotypes of the 20 species of the montium subgroup studied. All species showed the basic pattern of metaphase karyotype of the melanogaster species group, viz., one pair of sex chromosomes, two pairs of large V-shaped autosomes and one pair of dot-like 4th chromosomes. Results reported by Clayton and Wheeler (1975), although 22 species could be compared, were basically consistent with the present study. Changes in metaphase chromosome configurations of these species are entirely due to the process of acquisition of extra heterochromatin particularly involving the 4 th and/or Y chromosomes and to a lesser extent in the $\mathrm{X}$ chromosome. Interspecific variation in metaphase figure can be described as follows (see Table 1):

\section{$X$ chromosome}

The general configuration of the $\mathrm{X}$ chromosome is submetacentric ( $\mathrm{J}$-shaped) comprising one short arm which is totally heterochromatic and one long arm which contains mainly euchromatin. Metaphase variation has been observed in the short arm of the $\mathrm{X}$ chromosome of $D$. baimaii, D. mayri and $D$. pseudomayri which generally show a large submetacentric (LJ) shape. Apparently, the short arm of the X chromosome found in the latter two sibling species is relatively longer than that of $D$. baimaii which in turn contains more heterochromatin than that of the normal type (Figs. 6, $7,8)$. This feature of the $\mathrm{X}$ chromosome serves as a diagnostic cytological characteristic 


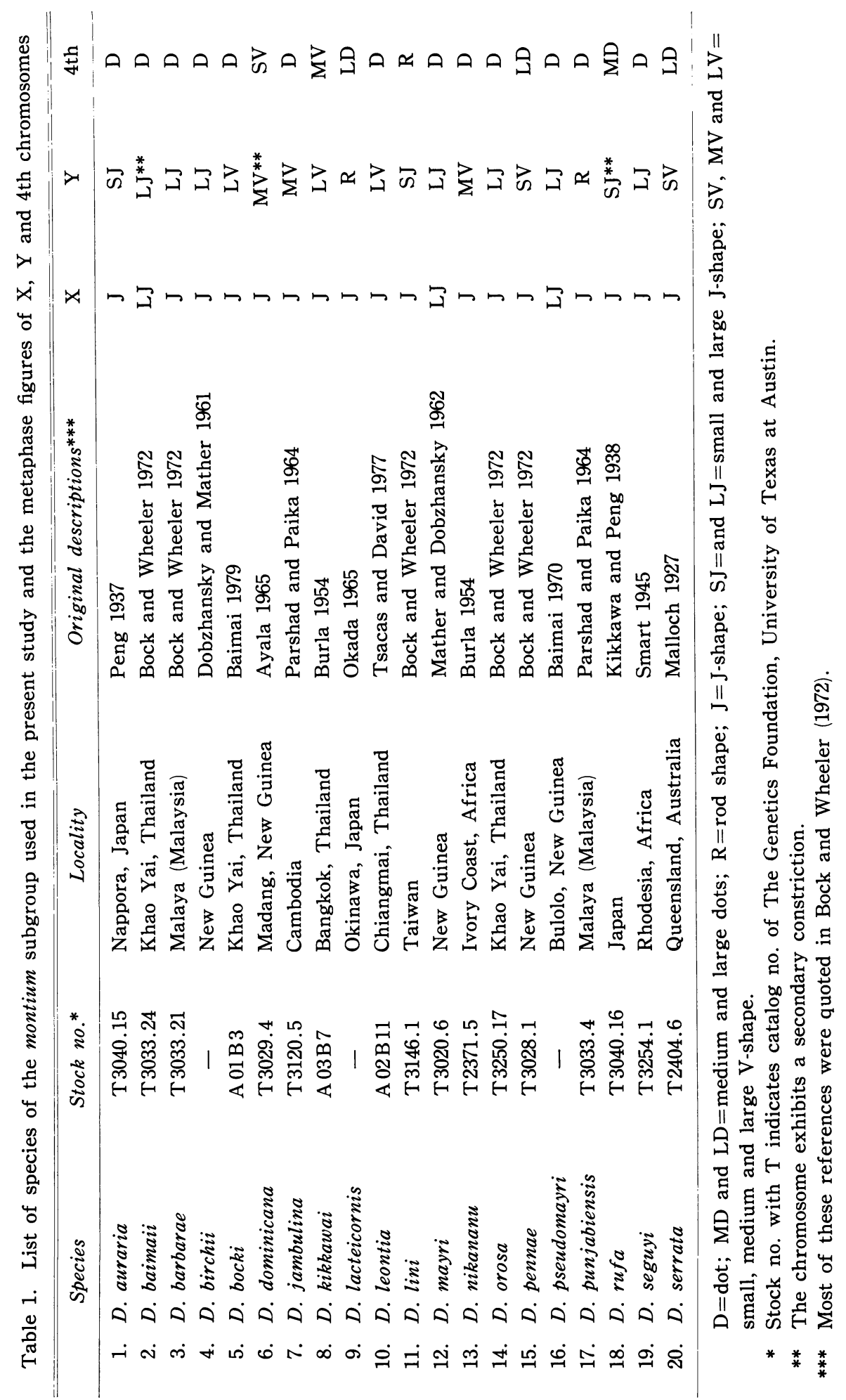


for these species. It seems, therefore, that the $\mathrm{X}$ chromosome is more uniform with respect to the acquisition of the amount of heterochromatin than the $\mathrm{Y}$ and 4 th chromosomes in the montium subgroup.

\section{$Y$ chromosome}

Among the 20 species of the montium subgroup in the present investigation, the most striking interspecific variation in metaphase has been encountered in the $Y$ chromosome which is, as a general rule, almost entirely heterochromatic. The pattern of $\mathrm{Y}$ chromosome variation of both size and shape is, of course, due to the different amount of heterochromatin. Six types of Y chromosomes can be recognized and simply described as follows:

1. Rod shaped (acrocentric or R) chromosome is the smallest and hence the most simple form of $\mathrm{Y}$ chromosome configuration. This type has been detected in two species, i.e. D. punjabiensis and D. lacteicornis (Figs. 1, 17).

2. Small J-shaped (submetacentric or SJ) Y chromosome has been encountered in D. auraria and $D$. lini (Figs. 2, 18). This type of $\mathrm{Y}$ chromosome obviously differs from the rod shape by the acquisition of an extra heterochromatic portion in the short arm. The Y chromosome of $D$. rufa is also regarded as a small $\mathrm{J}$-shape but with a secondary constriction occurring at the middle of the long heterochromatic arm (Fig. 14). Hence the $Y$ chromosome in this case appears to consist of 3 approximately equal segments of heterochromatin. It can be easily recognized and has been consistently observed in most preparations.

3. Large J-shaped $\mathrm{Y}$ chromosome (LJ) has been found in D. barbarae, D. orosa and D. seguyi (Figs. 3, 4, 5). Obviously, this type of $\mathrm{Y}$ chromosome is comparatively larger than the small J-shape particularly in the long heterochromatic arm. However, the J-shaped $\mathrm{Y}$ chromosome of $D$. baimaii consistently exhibits a secondary constriction at the middle of the long arm (Fig. 6). Moreover, D. mayri and D. pseudomayri each manifests a considerably larger J-shaped Y chromosome than that of the four species mentioned above. Apparently, the long arm of $\mathrm{Y}$ chromosome found in D. mayri contains relatively more heterochromatic material than that of the other species of this category (Fig. 7) while the short arm remains similar to that of the others. On the other hand, the short arm of the Y chromosome observed in D. pseudomayri consists of relatively more heterochromatin than that of other species of the same category (Fig. 8) while the long arm is apparently similar to that of the others. Thus it is interesting to note that these two sibling species which are sympatric in certain populations in New Guinea are recognizably different in the $\mathrm{Y}$ chromosome. This difference could be the result of a pericentric inversion or simply the acquisition of extra heterochromatin in the ancestral $\mathrm{Y}$ chromosome. This is not an uncommon phenomenon in animals especially in insects (White 1973).

Figs. 1-20. Photomicrographs depict larval metaphase chromosome configurations of the 20 species examined in this study. Each figure shows (a) female, (b) male (the Ychromosome is indicated by an arrow) and (c) diagramatic representation of the male metaphase karyotype (the heterochromatic portions are indicated in black). Scale: $5 \mu \mathrm{m}$. 

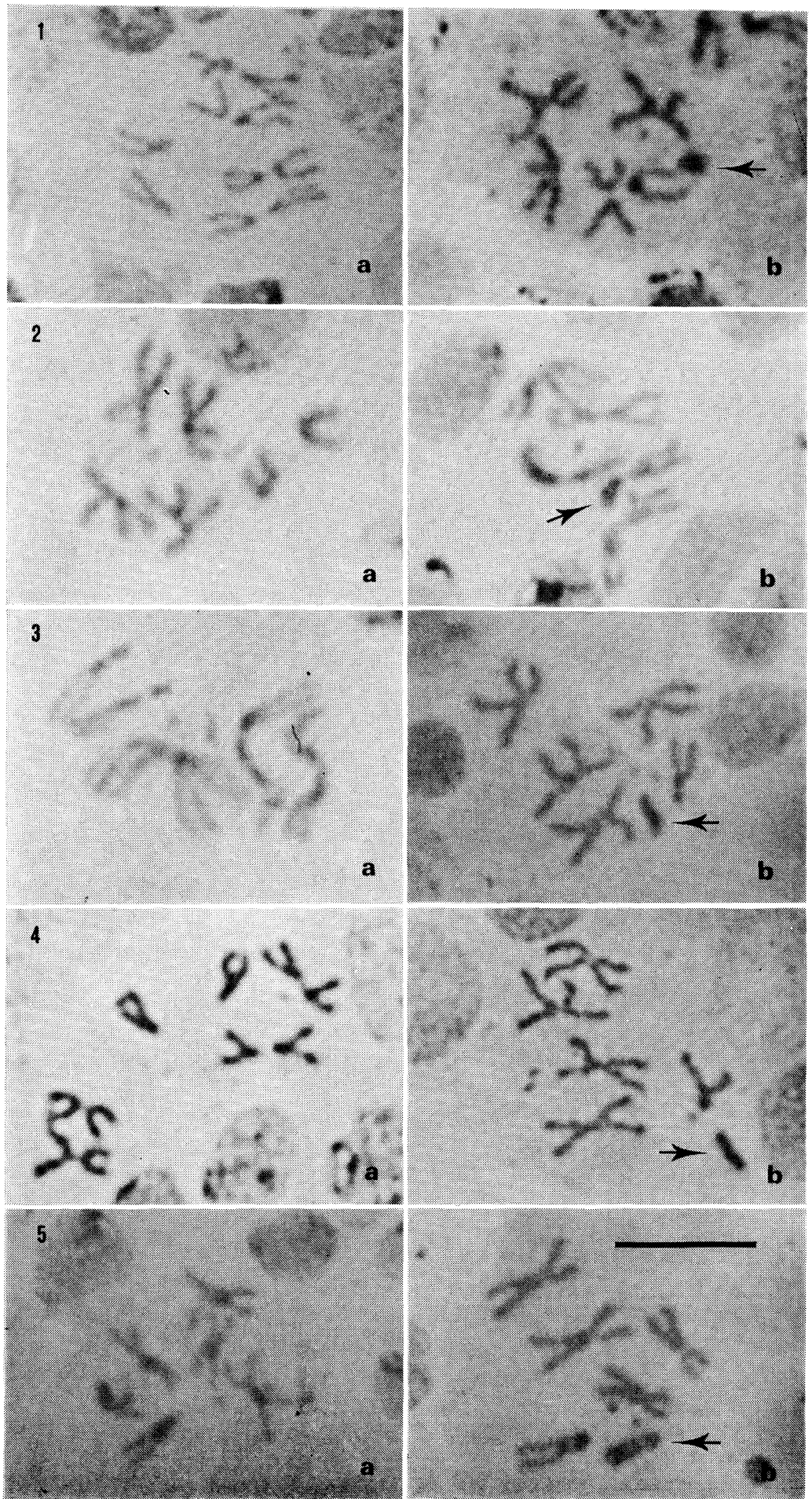
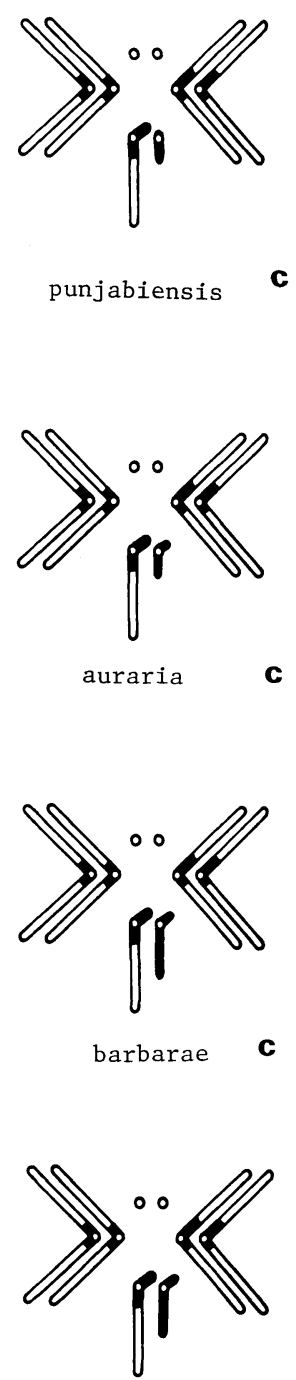

orosa c

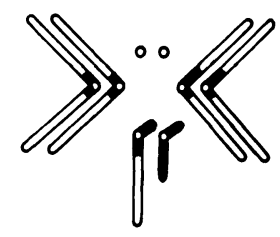

seguyi c

Figs. 1-5. 

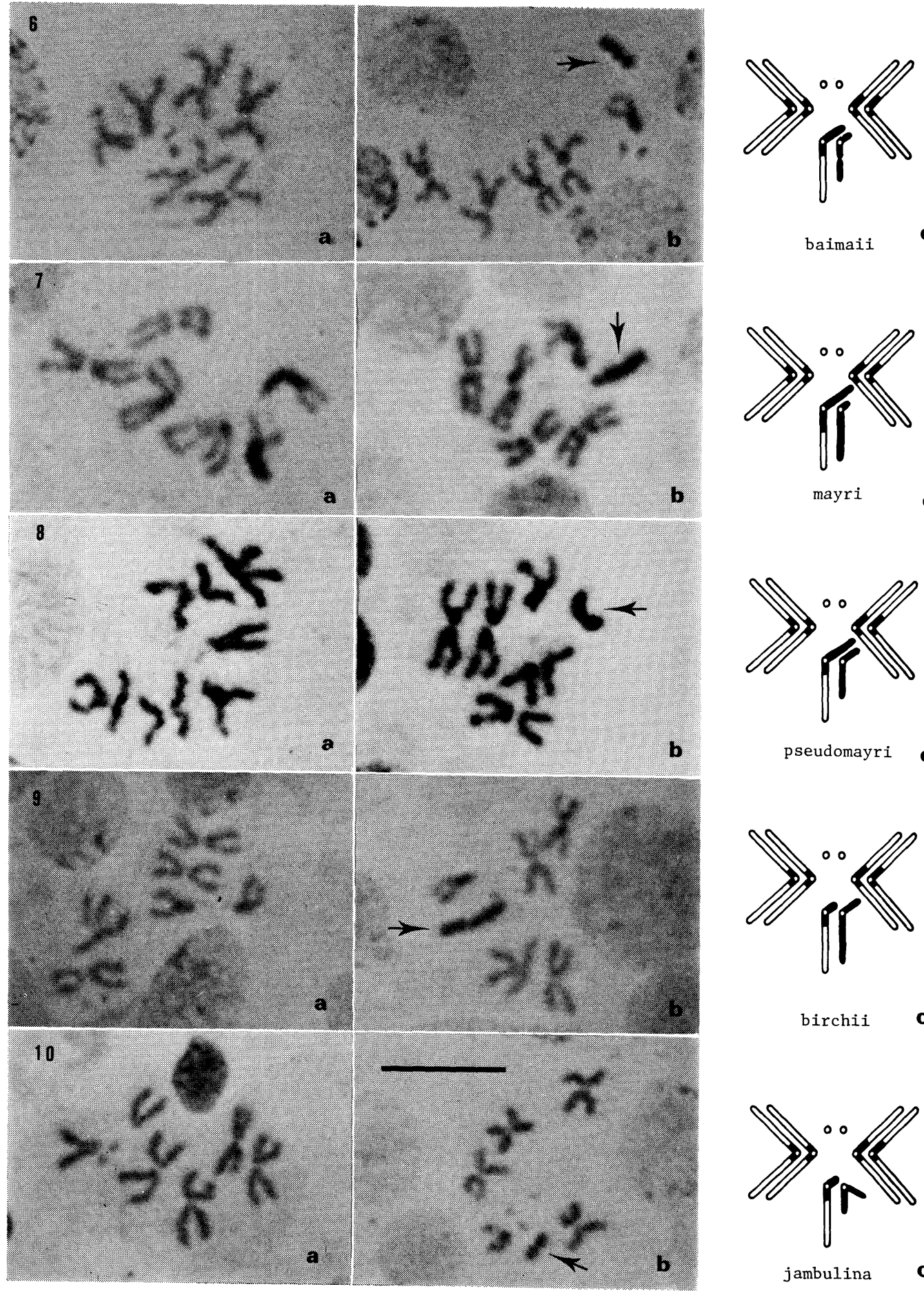

baimaii

c
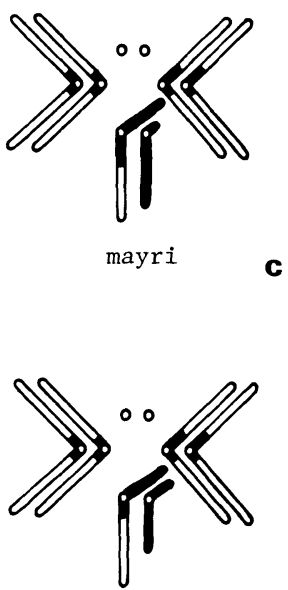

pseudomayri

c

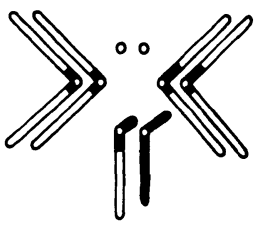

birchii c

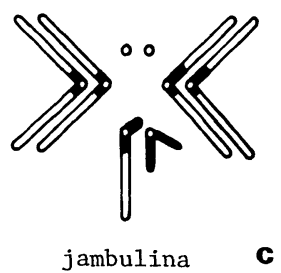

Figs. 6-10. 

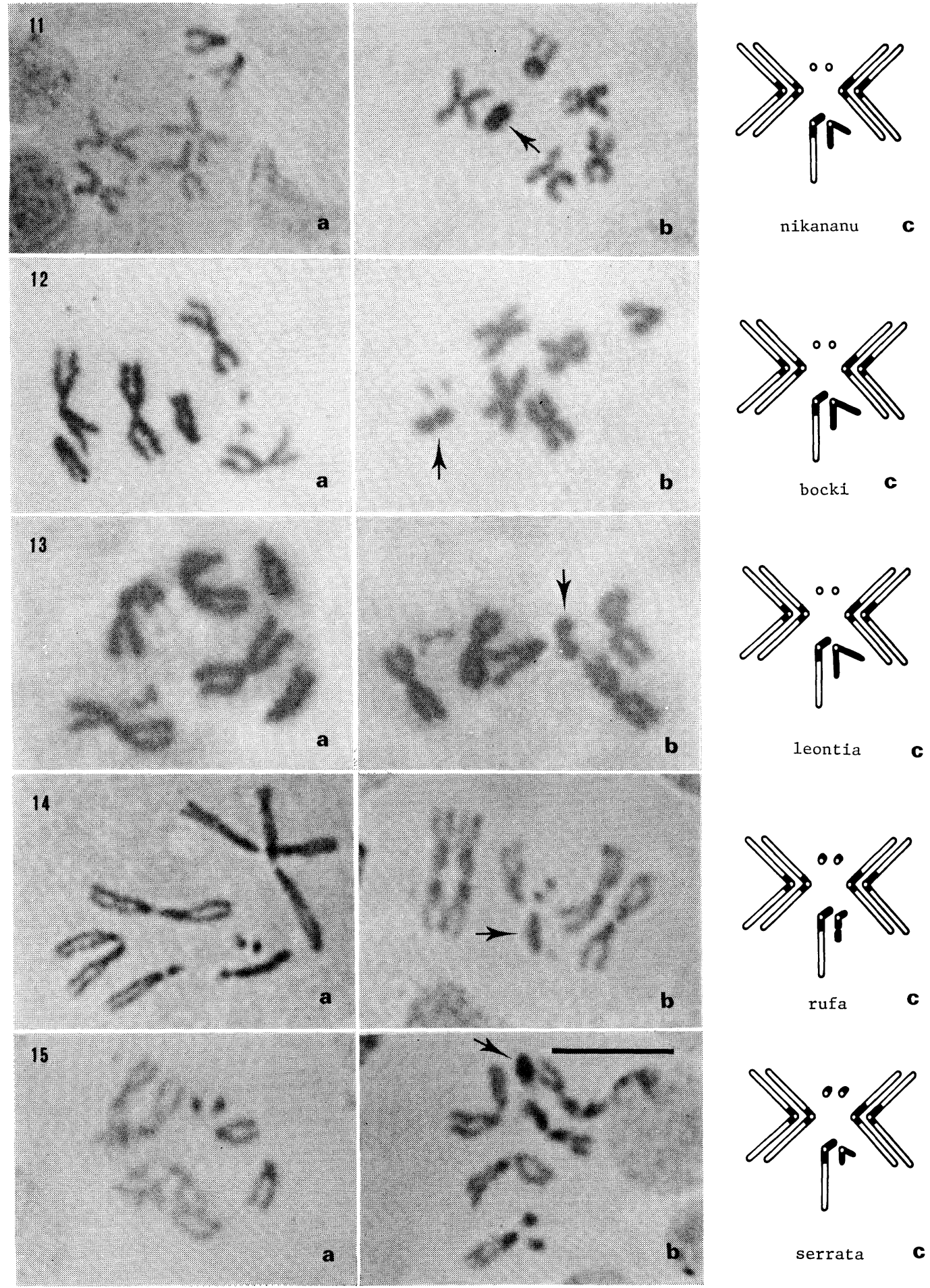

Figs. 11-15. 


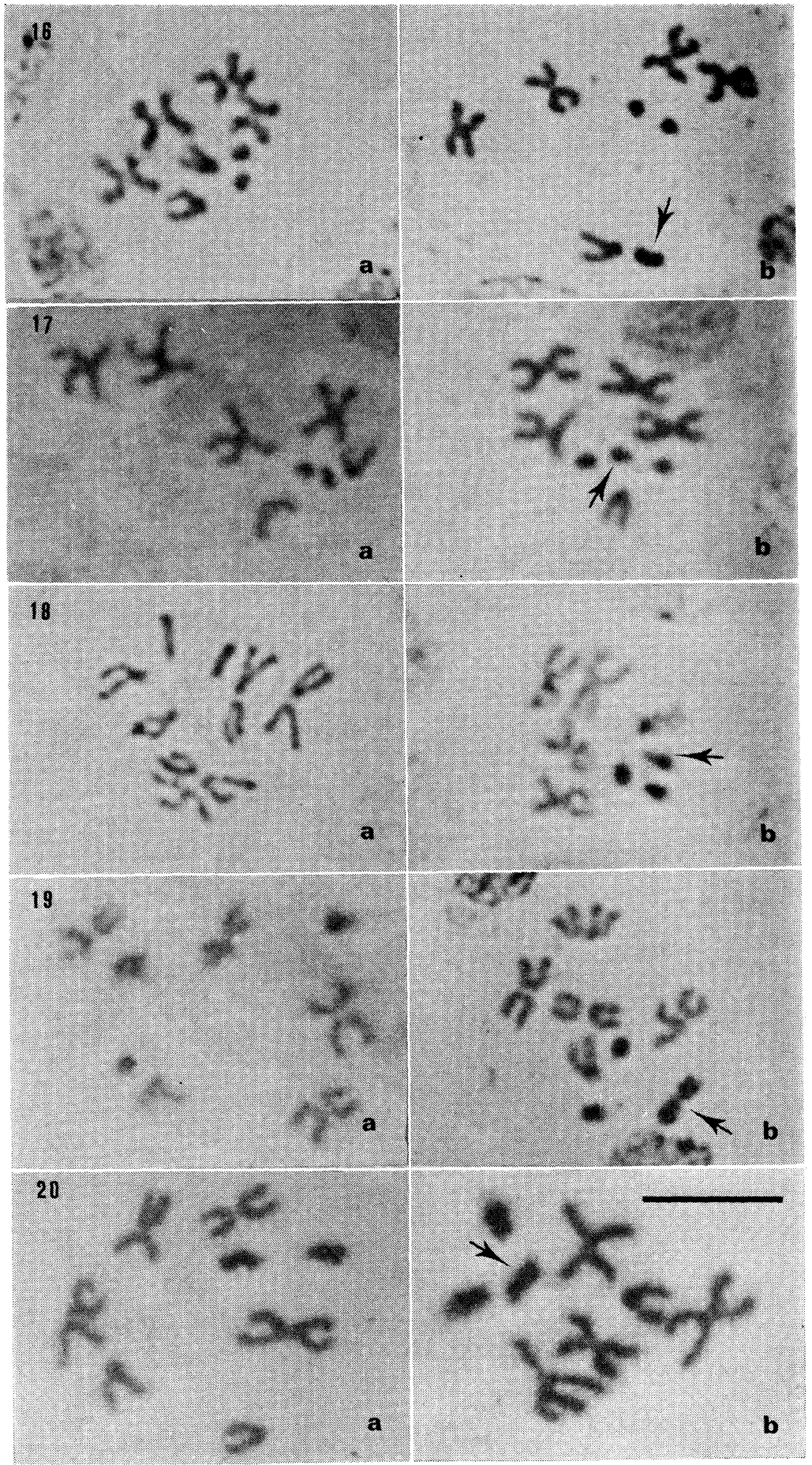

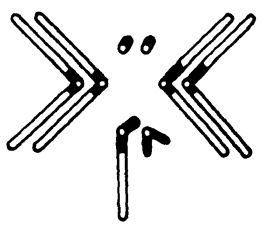

pennae c

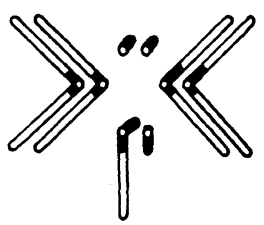

1acteicornis

c
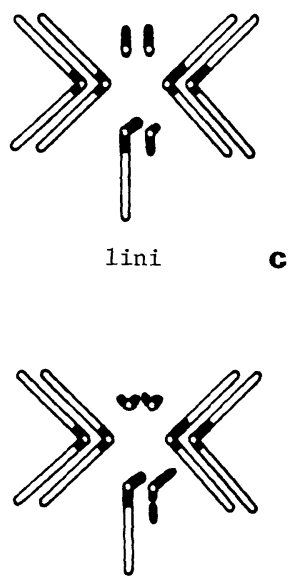

dominicana c

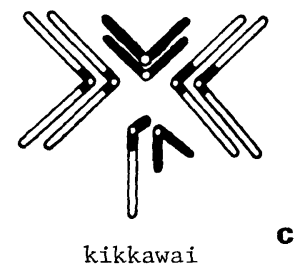

Figs. 16-20. 
A relatively larger $\mathrm{J}$-shaped $\mathrm{Y}$ chromosome has been observed in a strain of $D$. birchii from New Guinea as shown in Fig. 9. Three types of $\mathrm{Y}$ chromosome have been previously reported by Baimai (Baimai 1969a).

4. Small V-shape (metacentric or SV). This type of $\mathrm{Y}$ chromosome has been observed in two species viz. D. serrata and D. pennae (Figs. 15, 16). Because of its inconspicuous centromere and short length, this type of $\mathrm{Y}$ chromosome frequently appeared as a short rod in most metaphase preparations. However, the small V-shaped configuration was occasionally observed in some preparations. Furthermore, these two species also exhibit a large dot-shaped 4 th chromosome. Thus in some preparations, the $\mathrm{Y}$ and 4 th chromosomes could not be distinguished.

5. Medium V-shape (MV). This type of $\mathrm{Y}$ chromosome has been found in $D$. jambulina and D. nikananu (Figs. 10,11). The centromere position is normally apparent in most preparations. D. dominicana also manifests the medium V-shaped $\mathrm{Y}$ chromosome. However, it contains a unique secondary constriction in one arm. This feature together with the characteristic small V-shaped 4th chromosome make $D$. dominicana cytologically distinguishable from other species of the subgroup studied thus far (Fig. 19).

6. Large V-shape (LV). This type of $\mathrm{Y}$ chromosome is predominant among the D. kikkawai complex species. Thus it has been encountered in D. bocki, D. leontia and D. kikkawai (Figs. 12, 13, 20). D. kikkawai is one of the most remarkably variable species with respect to metaphase chromosomes (Baimai and Chumchong 1980).

\section{The 4th chromosome}

The normal dot-shape (D) is a common feature of the 4th chromosome (microchromosome) in most species of the montium subgroup. However, several species manifest deviation from the usual dot due to the acquisition of different amounts of extra heterochromatin at either side of the centromere. Thus $D$. rufa shows considerably more extra heterochromatin than the normal dot, transforming it into a medium size dot (designated as MD) (Fig. 14). Moreover, the 4 th chromosomes of $D$. serrata, $D$. pennae and D. lacteicornis contain considerably larger amounts of extra heterochromatin, transforming them into conspicuously large dots (Figs. 15, 16, 17). It is very difficult, if not impossible, to determine definitely the amount and location of extra heterochromatin added to the centromere. Therefore it is best to interpret such 4 th chromosomes as a large dot-shape (LD).

Even more remarkably, D. lini clearly exhibits an acrocentric 4 th chromosome (rod shape or R). This is a unique feature of $D$. lini that can be used as a cytologically diagnostic character for the species (Fig. 18). Furthermore, D. dominicana apparently manifests a very small metacentric 4 th chromosome (small V-shape, SV) which contains a small portion of heterochromatin on each side of the centromere (Fig. 19). However, such a V-shaped configuration has been occasionally observed. Generally, it appears as a very large dot 4 th chromosome in most preparations because of its relatively short heterochromatic arms. In such a case, the 4 th chromosome of $D$. dominicana appeared as a large dot similar to those of the other 3 species described above.

D. birchii has been shown to have two types of 4 th chromosome (Baimai 1969a). 
The normal dot shape as figured in this paper is the most common form while the Jshape is restricted to certain populations in New Guinea.

With respect to the 4 th chromosome configuration, D. kikkawai is the most variable species recorded thus far in this subgroup or even in the genus Drosophila (Baimai and Chumchong 1980). This species exhibits at least six types of 4th chromosome each of which seems to have a definite pattern of geographic distribution. Fig. 20 shows the medium V-shaped 4th chromosome of D. kikkawai of the Bangkok strain which is apparently very common in the Oriental region. Such variation in the 4 th chromosome due entirely to different amounts of heterochromatin makes $D$. kikkawai one of the most interesting species with respect to metaphase karyotype.

Although phylogenetic relationships within the montium subgroup are poorly known, the few groups of closely related species that have been investigated thus far exhibit two cytotaxonomic trends. In one direction, certain groups of species, for example, mayri-pseudomayri and bocki-leontia, show similarity in both morphology (especially male genitalia) and metaphase karyotype. On the contrary, certain groups of taxonomically most closely related species appear to be karyotypically quite distinct, viz. birchii-dominicana-serrata, and leontia-kikkawai. The same situation have been observed in certain groups of homosequential species of Hawaiian Drosophila (Carson et al. 1970; Carson and Kaneshiro 1976; Baimai and Ahearn 1978). The evidence seems to suggest that the process of acquisition of heterochromatin has, in part, played a role in the evolution of some groups of these taxa. The degree of metaphase variation due mainly to different amounts of heterochromatin may remain to be discovered in this large species subgroup. It is hoped that future investigations of cytotaxonomy may be useful in certain groups of these taxa.

\section{ACKNOWLEDGMENTS}

I thank Dr. I. R. Bock for critical reading of the manuscript and Mrs. C. Chumchong for maintaining the stocks for his study. Most of the culture stocks used in this study were kindly provided by Prof. M. R. Wheeler, University of Texas.

\section{LITERATURE CITED}

Baimai, V., 1969a Karyotype variation in Drosophila birchii. Chromosoma (Berl.) 27: 351-394.

Baimai, V., 1969b Karyotype variation in D. montium. Drosoph. Inf. Serv. 44: 115-117.

Baimai, V., 1977 Chromosomal polymorphisms of constitutive heterochromatin and inversions in Drosophila. Genetics 85: 85-93.

Baimai, V., 1978 Karyotypical variation in the Drosophila kikkawai species complex. Inter. Congr. Genet. Part I: 245.

Baimai, V., and J. N. Ahearn, 1978 Cytogenetic relationships of Drosophila affinidisjuncta Hardy. Amer. Midl. Nat. 99: 352-360.

Baimai, V., 1979 A new species of Drosophila kikkawai complex from Thailand (Diptera: Drosophilidae). Pacific Insects 21: 235-240.

Baimai, V., and C. Chumchong, 1980 Metaphase karyotype variation and the geographic distribution of the three sibling species of the Drosophila kikkawai complex. Genetica (in press). 
Bock, I. R., and M. R. Wheeler, 1972 The Drosophila melanogaster species group. Univ. Texas Publ. 7213: 1-102.

Carson, H. L., and K. Y. Kaneshiro, 1976 Drosophila of Hawaii: Systematics and ecological genetics. Ann. Rev. Ecol. Syst. 7: 311-345.

Carson, H. L., D. E. Hardy, H. T. Spieth, and W. S. Stone, 1970 The evolutionary biology of the Hawaiian Drosophilidae. In "Essays in Evolution and Genetics in Honor of Theodosius Dobzhansky” (M. K. Hecht, and W. C. Steere, eds.) pp. 437-543. Appleton-Century-Crofts, New York.

Clayton, F. C., and M. R. Wheeler, 1975 A catalog of Drosophila metaphase chromosome configurations. In "Handbook of Genetics" (R. C. King, ed.) Vol. 3, 471-512. Plenum Press, New York.

Lewis, E. B. and L. S. Riles 1960 A new method of preparing larval ganglion chromosomes. Drosoph. Inf. Serv. 34: 118-119.

Tsacas, L., and J. David, 1977 Systematics and biogeography of the Drosophila kikkawai complex, with descriptions of new species (Diptera: Drosophilidae). Annls. Soc. Ent. Fr. (N. S.) 13: 675-693.

White, M. J. D., 1973 “Animal Cytology and Evolution” 3rd ed. Cambridge Univ. Press, London. 